\title{
Comparative Study between Fibro-Test and Egy-Score as Non-Invasive Markers for Assessment of Hepatic Fibrosis in Chronic Hepatitis C
} Fathy Ghamry Abd El razek Elghmary ${ }^{1}$, Hosni Abd El Kariem Younis ${ }^{1}$, Mohammed Mahmoud Noser ${ }^{1}$, Ayman Abd Al Aziz Abd Al Rahman', Mohammed Yousri Shaheen ${ }^{2}$ \& Ahmed Mohammed Mostafa ${ }^{*}$

Departments of ${ }^{1}$ Internal Medicine and ${ }^{2}$ Clinical Pathology,Faculty of Medicine, Al-Azhar University *Corresponding author: Ahmed Mohammed Mostafa, Mobile: 002010666866572, E-Mail: ahmedmostafa94@gmail.com

Abstract

Backgound: Liver fibrosis (LF) occurs in response to almost all causes of chronic liver injury. Assessing LF is important for both predicting disease progression and monitoring efficacy of therapeutic measures. Most noninvasive tests of liver fibrosis were developed with the aim of discriminating between "insignificant", (F0-F1) by METAVIR and clinically "significant" fibrosis ( $\geq$ F2) by METAVIR or for identifying or excluding established cirrhosis in patients with well compensated chronic liver disease. Both these aims are clinically the most relevant. Aim: We aimed to compare the diagnostic accuracy of FibroTest and Egy-Score as predictors of stage of hepatic fibrosis in a prospectively enrolled cohort of Egyptian patients with chronic hepatitis C. Patients and Methodlogy: Twenty patients, treatment naïve chronic hepatitis $\mathrm{C}$ patients were enrolled. They were 16 males $(80 \%)$ and 4 females (20\%) mean age of these patients was $53.55+14.3$ (rang 18 73 years). The study was carried out in the Department of Gastroenterology and Hepatology, Elhussin hospital, Al-Azhar University during the period between March 2016 and March 2018. Results: Our results showed a highly significant positive correlation between stage of hepatic fibrosis by METAVIR and fibrotest. Our results showed a highly significant positive correlation between stage of hepatic fibrosis by METAVIR and EGY-SCOR. Our scores depend mainly on simple routinely used laboratory parameters (total bilirubin, albumin, platelet count) in addition to age and 2 non routine tests (CA 19-9 and Alpha-2Macroglobulin). Although this panel needs to be done in validated laboratories, the cost of our score is much cheaper than other well-known and patented tests such as FibroTest and the net results of both methods nearly the same. Conclusion: Egy-Score can be applied easily in clinical practice to exclude severe hepatic fibrosis/cirrhosis in patients with contraindication for liver biopsy or those who are reluctant to do it. Egy-score would need further valida $\neg$ tion to be regarded as an alternative to liver biopsy. Recommendations: Physician should be careful when interpreting elevated levels of tu $\neg$ mor markers CA 19-9 and CA 125 in patients with chronic liver disease as this could be a benign elevation related to hepatic fibrosis and not necessarily due to underlying malignancies. Elevation of the tumor markers such as CA19.9 have been associated with cholestasis in liver disease patients and this may give false positive results for our scores which give Limitations to our study.

Keyowrds: Fibro-Test, Egy-Score, Chronic Hepatitis C

\section{Introduction}

Liver fibrosis (LF) occurs in response to almost all causes of chronic liver injury. Assessing LF is important for both predicting disease progression and monitoring efficacy of therapeutic measures ${ }^{(1)}$.

Invasive diagnosis using liver biopsy with histological examination is most commonly used as reference standard for the assessment of fibrosis but is hampered by several disadvantages: large sampling error, consistent inter-observer disagreement, high emotional cost of patient and enormous health care commitment in case of rare but possible severe complications ${ }^{(2)}$.

Thus, noninvasive methods of measuring the degree of hepatic fibrosis have been developed, such as surrogate serum fibrosis markers, liver stiffness measurement using FibroScan (Echosense, Paris, France) ${ }^{(3)}$, various imaging methods and glycomics ${ }^{(4)}$. Biochemical markers of LF, because they can be tested noninvasively, reproducibly, and reliably, may constitute a true alternative to liver biopsies ${ }^{(5)}$.

Several noninvasive direct and indirect serum markers, capable to predict the presence of significant fibrosis or cirrhosis in patients with chronic liver disease have been reported (6).

Fibro-Test is considered the most widely used and validated noninvasive test for assessment of hepatic fibrosis; especially in chronic hepatitis $\mathrm{C}^{(2)}$.

Fibro-Test is composed of 6 
parameters

(alpha2-macroglobulin, haptoglobin, gamma glutamyl transpeptidase, age, bilirubin, Apo-lipoprotein Al, and sex) in a regression equation and is patented for a French company (BioPredicitve) and costs around 50 euros per test ${ }^{(7)}$.

Recent local studies showed the usefulness of a new non-patented panel of biomarkers "Egy-Score" as predictor for severe hepatic fibrosis in different chronic liver diseases as well as in Egyptians with chronic hepatitis $\mathrm{C}^{(\boldsymbol{8})}$.

Egy-Score is calculated using a regression equation depends on the serum levels of CA19-9, age, alpha-2-macroglobulin, total bilirubin, platelet count and albumin. EgyScore could discriminate early or no hepatic fibrosis (F0-F2 METAVIR) from severe hepatic fibrosis (F3-F4 METAVTR) with overall accuracy of $83.7 \%$. Egy-Score can be calculated using the following equation: EgyScore $=3.52+0.0063 \times$ CA19-9 $+0.0203 \times$ age $+0.4485 \mathrm{x}$ alpha 2 macroglobulin $+0.0303 \mathrm{x}$ bilirubin $-0.0048 \mathrm{x}$ platelet $-0.0462 \mathrm{x}$ albumin (9).

Aim of the Work

We aim to compare the diagnostic accuracy of FibroTest and Egy-Score as predictors of stage of hepatic fibrosis in a prospectively enrolled cohort of Egyptian patients with chronic hepatitis $\mathrm{C}$.

Patients and Methods

Patients:

Twenty patients, treatment naïve chronic hepatitis $\mathrm{C}$ patients were enrolled. They were 16 males (80\%) and 4 females (20\%) mean age of this patients was $53.55+14.3$ (rang 18 73 years).The study was carried out in the department of gastroenterology and hepatology, Elhussin hospital, Al-Azhar University during the period between March 2016 and March 2018.

\section{Exclusion criteria:}

Patients with other types of chronic liver disease e.g., HBsAg, autoimmune hepatitis, Bilharziasis and treated patients were excluded.

\section{Methods:}

All patients were subjected to:

1- History taking included: symptoms of hepatobiliary disorder (eg; yellowish discolouration of eyes, itching, abdominal or lower limb swelling, fatigue, dark urine, etc) and dates of possible exposure to viral infection or onset of hepatitis when known. History of other autoimmune disorders, other chronic liver diseases or previous antiviral or immunosuppressive therapy.

\section{2- Clinical assessment:}

General examination: Vital signs including pulse, blood pressure, temperature and respiratory rate, general examination for manifestations of liver cell failure as jaundice, palmer erythema, spider naevi, flapping tremors and oedema of both lower limbs etc.

Local examination: Abdominal examination for detection of hepatosplenomegaly, ascites or other manifestations of liver disease.

3- Laboratory tests: Including

- Routine laboratory evaluation: urine, stool, complete blood counts, renal functions.

- Liver function tests; alanine aminotransferase (ALT), aspartate aminotransferase (AST), total and direct bilirubin, INR (international normalized ratio), albumin and platelet count and total protein.

- HCVAbs, HBsAg, ANA, Bilharzial agglutination titer.

- Fibrotest (gamma glutamyl transpeptidase (GGT), serum apolipoproteinA1 (Apo-A1), Alpha2_macroglobulin, haptoglobin, bilirubin, Age and sex) test done in a validated laboratory and calculated through Biopredictive website (http:// biopredictive.com).

- Egy-score test parameters (CA19.9, age, Alpha2_macroglobulin, total bilirubin, albumin and platelet count). Egy-score calculated by the following formula:

- Egyscore $=3.52+0.0063 *$ CA19.9+0.0203* Age $+0.4485^{*} \quad$ Alpha2 macroglobulin $+0.0303 *$ bilirubin$0.0048^{*}$ platelet count-0.0462* albumin.

\section{4- Abdominal ultrasound:}

- Abdominal and pelvic ultrasound were done for all subjects (using Philips iU22 x MATRIX Ultrasound system) to assess the severity of liver disease and to rule out presence of hepatic, pancreatic or ovarian lesions. Cases suspected to have any lesions that could be malignant were referred for further 
evaluation by dynamic contrast enhanced imaging studies to rule out presence of malignancy.

5- Liver biopsy and histopathological assessment:

- Liver biopsy was performed by using the 18 gauge Surecut liver biopsy needle (modified Menghini liver aspiration needle), using the subcutaneous intercostal approach. Prior to liver biopsy informed consent was obtained, and blood CBC, PT/INR were done.

- Ultrasound abdomen was carried out to mark the biopsy site, and to rule out any anatomical abnormality of the gall bladder, or silent lesion (such as haemangioma) within the liver parenchyma. Similarly chest X-ray was done to rule out Chiladiti Syndrome (the interposition of bowel between the inner intercostal wall and liver parenchyma). All biopsy material slides were stained with haematoxylin and eosin and reticulin stains. The biopsies were interpreted by qualified histopathologists (prof.mohmmed yosri shaheen professor of clinical pathology in Elhussin Hospital Al Azhar University).

- The liver biopsy was scored according to the METAVIR scoring system with five stagesfor fibrosis ${ }^{\mathbf{( 1 0 )}}$ :

F0 no fibrosis

F1 portal fibrosis without septa

F2 portal fibrosis with few septa

F3 numerous septa without cirrhosis F4 cirrhosis

- Necro-inflammatory lesions, was graded as follows:

\section{A0 no histological activity}

A1 mild activity

A2 moderate activity

A3 severe activity.

- Informed and written consent was taken from each participant in the study.

Statistical analysis of data and developing predictive:

MODEL:

After collecting date, we made cleaning for data by examining minimum and maximum values in each variable to detect any abnormal or outlier value.
Then we summarized data using descriptive statistics (mean \& SD for continuous variables, and number \& percentage for categorical).

We examined different cutoff points for each variable (ex. CA 19-9) to get the strongest one.

Then we checked correlations and predictive values between several variables and outcome.

Previous steps will give us a good idea about importance of variables.

Statistics were done by computer using Epi - info. Software, version 6.04. A word processing, database and statistics program. The tests used were:

1) $\mathrm{X}$ mean, SD standard deviation: to measure the central tendency of data and the distribution of data around their mean value.

2) Student's t test: for testing statistical significant difference between mean values of two samples.

3) Kurskell- Wallis test : to test for statistical significant relation between different variable or grades in qualitative data.

4) ANOVA or F test: to test for significant difference between more than two samples mean values.

5) Pearson correlation coefficient test: (r) to test for linear relation between two numeric variables.

6) Kurskell- Wallis test: non parametric test for comparing two groups of data not normally distributed or for small sample size.

7) Multiple regressions: to analyze a single dependent variable $Y$ that is of interest to predict one or more independent variables $\mathrm{X}$ that explain the variations that occur in (Y).

8) Kurskell- Wallis test: for comparing two independent proportions when the expected observation in any cell.

Significant result is considered if $\mathrm{p}<0.05$. Highly significant result is considered if $\mathrm{p}<$ 0.01

Predictive value positive (PVP): of a diagnostic test is the probability that an individual with a positive test result has a disease. It is also the proportion of diseased individuals in relation to individuals with a positive test result.

$$
P V P=a /(a+b) \times 100
$$


PVP = diseased with positive test / all with positive screening test $\mathrm{x} 100$

Predictive value negative (PVN): of a diagnostic test is the probability that an individual with a negative test result does not have the disease. It is also the proportion of disease free individuals in the population of individuals with negative screening test result.

$$
\mathrm{PVN}=\mathrm{d} /(\mathrm{c}+\mathrm{d}) \times 100
$$

Results

Tweenty patients (16 male and 4 female) were included in this study. The mean age of these patients was $53.55+-14.3$ (rang $18 \_73$ years) all of them treatment naïve with chronic liver disease of hepatitis C etiology.

Liver biopsy was taken from each patient for histopathological examination and classify according to metavir scoring and the results show:

- Three patients were F1 (15\%)

- Three patients were F2 (15\%)

- Five patients were F3 (25\%)

- Five patients were F4 (25\%)

- Four patients were F5 (20\%)

Distribution of study patients as regard stage of fibrosis measured by fibrotest show (15\% F1, 15\% F2, 25\% F3, 25\% F4 and 20\% F5).

Comparison between study patient as regard age, GGT, serum bilirubin, serum haptoglobin based on stage of fibrosis measured by fibrotest and metavir showed significant positive correlation between two parameter (table $2,4,6$ ) respectively.

Comparison between study patient As regard ALT, alpha2 macroglobulin, CA19_9, Egy-score based on stage of fibrosis measured by fibrotest and metavir showed high significant positive correlation between two parameter ( table $3,10,11,12$ ) respectively .

On the other hand Comparison between study patient As regard serum albumin, platelet based on stage of fibrosis measured by fibrotest and metavir showed significant negative correlation between two parameter (table 5, 7) respectively.

But Comparison between study patient As regard apo A1 based on stage of fibrosis measured by fibrotest and metavir showed no significant correlation between two parameter (table 8).

Table (1): Descriptive data of study patients :
Our results showed highly significant positive correlation between Egy-score and alpha2 macroglobulin, serum bilirubin, CA19_9 respectively.

On the other hand the results showed highly significant negative correlation between Egy-score and serum albumin, platelet count respectively.

But no significant correlation between Egy-score and ALT, apo A1, serum haptoglobin respectively.

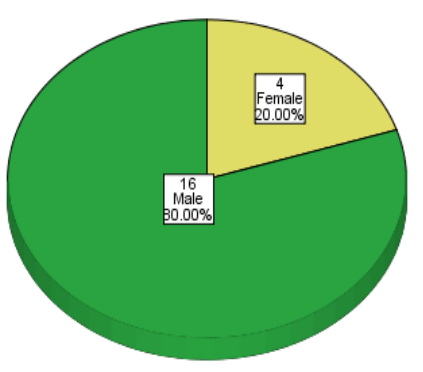

Figure (1): Distribution of study patients as regard sex.

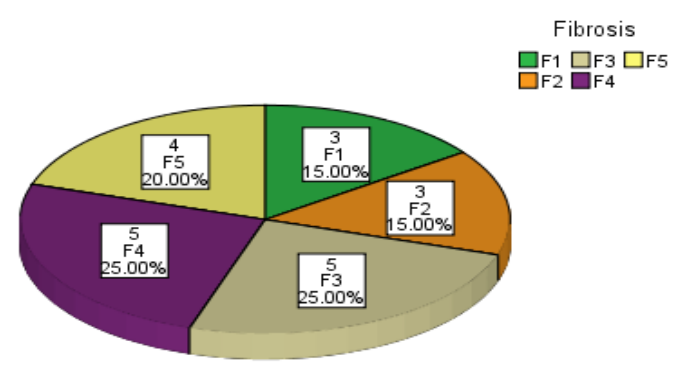

Figure (2): Distribution of study patients as regard stage of fibrosis measured by fibrotest.

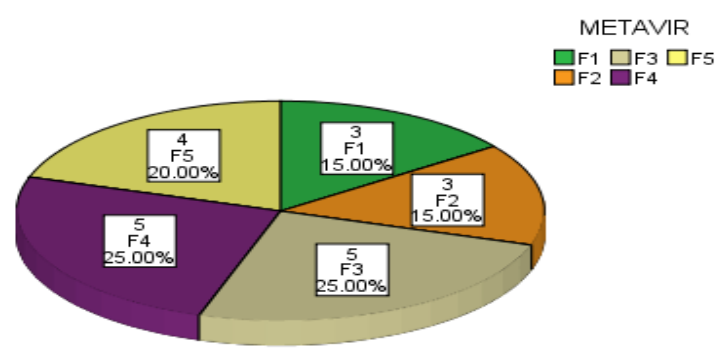

Figure (3): Distribution of study patients as regard METAVIR. 
Fathy Elghmary et al.

\begin{tabular}{|c|c|c|c|c|}
\hline & Minimum & Maximum & Mean & Std. Deviation \\
\hline Age (years) & 18 & 73 & 53.55 & 14.336 \\
\hline ALT (IU/dl) & 16 & 59 & 41.30 & 12.807 \\
\hline GGT (IU/dl) & 18 & 129 & 51.56 & 27.589 \\
\hline Serum albumin (mg/dl) & 30.0 & 50.8 & 41.425 & 5.5527 \\
\hline Serum bilirubin (mg/dl) & 3.8 & 24.0 & 12.704 & 5.8601 \\
\hline Platelet count (x109/L) & 115 & 368 & 242.30 & 86.553 \\
\hline apo A1 (mg/dl) & .86 & 2.02 & 1.2570 & .27832 \\
\hline Serum Haptoglobin (mg/dl) & .20 & 2.75 & 1.1145 & .57419 \\
\hline $\boldsymbol{\alpha 2 m a c r o g l o b u l i n ~ ( g / d l ) ~}$ & 1.02 & 4.50 & 2.3035 & .91441 \\
\hline CA19-9 (ml) & 1.5 & 35.0 & 16.895 & 10.1743 \\
\hline Egy-Score & .904690 & 4.982475 & 3.02563945 & 1.141213845 \\
\hline
\end{tabular}

Table (2): Comparison between study patient as regard age based on stage of fibrosis measured by fibrotest and METAVIR (by non-parametric Kruskal Wallis test):

\begin{tabular}{|c|c|c|c|c|c|c|}
\hline & & $\mathbf{N}$ & Mean & Std. Deviation & p-value & Significance \\
\hline \multirow[t]{5}{*}{ Age (years) } & $\mathrm{F} 1$ & 3 & 36.33 & 15.948 & \multirow{5}{*}{0.01} & \multirow{5}{*}{ S. } \\
\hline & $\mathrm{F} 2$ & 3 & 43.67 & 13.051 & & \\
\hline & $\mathrm{F} 3$ & 5 & 63.60 & 5.413 & & \\
\hline & $\mathrm{F} 4$ & 5 & 50.20 & 11.300 & & \\
\hline & F5 & 4 & 65.50 & 6.137 & & \\
\hline
\end{tabular}

Table (3): Comparison between study patient as regard ALT based on stage of fibrosis measured by fibrotest and METAVIR (by non-parametric Kruskal Wallis test):

\begin{tabular}{|c|c|c|c|c|c|c|}
\hline & & $\mathbf{N}$ & Mean & Std. Deviation & p-value & Significance \\
\hline \multirow[t]{5}{*}{ ALT (IU/dl) } & $\mathrm{F} 1$ & 3 & 25.67 & 14.224 & \multirow{5}{*}{$<0.01$} & \multirow{5}{*}{ H.S. } \\
\hline & $\mathrm{F} 2$ & 3 & 51.00 & 2.000 & & \\
\hline & F3 & 5 & 52.80 & 6.017 & & \\
\hline & $\mathrm{F} 4$ & 5 & 30.00 & 3.162 & & \\
\hline & F5 & 4 & 45.50 & 6.658 & & \\
\hline
\end{tabular}

Table (4): Comparison between study patient as regard GGT based on stage of fibrosis measured by fibrotest and METAVIR (by non-parametric Kruskal Wallis test):

\begin{tabular}{|c|c|c|c|c|c|c|}
\hline & & $\mathbf{N}$ & Mean & Std. Deviation & p-value & Significance \\
\hline \multirow[t]{5}{*}{ GGT (IU/dl) } & $\mathrm{F} 1$ & 3 & 29.33 & 13.317 & \multirow{5}{*}{0.024} & \multirow{5}{*}{ S. } \\
\hline & $\mathrm{F} 2$ & 3 & 51.20 & 16.041 & & \\
\hline & F3 & 5 & 47.14 & 10.302 & & \\
\hline & $\mathrm{F} 4$ & 5 & 37.68 & 24.515 & & \\
\hline & F5 & 4 & 91.40 & 24.974 & & \\
\hline
\end{tabular}


Table (5): Comparison between study patient as regard serum albumin based on stage of fibrosis measured by fibrotest and METAVIR (by non-parametric Kruskal Wallis test):

\begin{tabular}{|c|c|c|c|c|c|c|}
\hline & & $\mathbf{N}$ & Mean & Std. Deviation & p-value & Significance \\
\hline \multirow{5}{*}{$\begin{array}{l}\text { Serum albumin } \\
(\mathrm{mg} / \mathrm{dl})\end{array}$} & $\mathrm{F} 1$ & 3 & 47.033 & 5.1228 & \multirow{5}{*}{0.011} & \multirow{5}{*}{ S. } \\
\hline & $\mathrm{F} 2$ & 3 & 44.333 & 3.7687 & & \\
\hline & F3 & 5 & 44.800 & 2.5846 & & \\
\hline & $\mathrm{F} 4$ & 5 & 36.940 & 4.6651 & & \\
\hline & F5 & 4 & 36.425 & 2.1515 & & \\
\hline
\end{tabular}

Table (6): Comparison between study patient as regard serum bilirubin based on stage of fibrosis measured by fibrotest and METAVIR (by non-parametric Kruskal Wallis test):

\begin{tabular}{|c|c|c|c|c|c|c|}
\hline \multirow{2}{*}{$\begin{array}{c}\text { Serum bilirubin } \\
\text { (mg/d) }\end{array}$} & & $\mathbf{N}$ & Mean & Std. Deviation & p-value & \multirow{2}{*}{ Significance } \\
\cline { 2 - 5 } & $\mathrm{F} 2$ & 3 & 7.300 & 1.4731 & & \\
\cline { 2 - 6 } & $\mathrm{F} 3$ & 5 & 11.060 & 4.9516 & \multirow{3}{*}{0.014} & \multirow{2}{*}{ S. } \\
\cline { 2 - 6 } & $\mathrm{F} 4$ & 5 & 16.296 & 5.0733 & & \\
\cline { 2 - 6 } & $\mathrm{F} 5$ & 4 & 18.350 & 4.7177 & & \\
\hline
\end{tabular}

Table (7): Comparison between study patient as regard platelet count based on stage of fibrosis measured by fibrotest and METAVIR (by non-parametric Kruskal Wallis test):

\begin{tabular}{|c|c|c|r|r|r|r|}
\hline & & N & Mean & Std. Deviation & p-value & Significance \\
\hline \multirow{3}{*}{$\begin{array}{c}\text { Platelet count } \\
(\times \mathbf{1 0 9 / L})\end{array}$} & F1 & 3 & 303.33 & 47.522 & & \\
\cline { 2 - 5 } & F2 & 3 & 308.00 & 95.394 & \\
\cline { 2 - 5 } & F3 & 5 & 235.00 & 72.111 & \multirow{2}{*}{0.036} & \multirow{2}{*}{ S. } \\
\cline { 2 - 5 } & F4 & 5 & 257.00 & 79.759 & & \\
\cline { 2 - 5 } & F5 & 4 & 138.00 & 38.245 & & \\
\hline
\end{tabular}

Table (8): Comparison between study patient as regard apo A1 based on stage of fibrosis measured by fibrotest and METAVIR (by non-parametric Kruskal Wallis test):

\begin{tabular}{|c|c|c|c|c|c|c|}
\hline & & $\mathbf{N}$ & Mean & Std. Deviation & p-value & Significance \\
\hline \multirow[t]{5}{*}{ apo $A 1(\mathrm{mg} / \mathrm{dl})$} & $\mathrm{F} 1$ & 3 & 1.2233 & .14224 & \multirow{5}{*}{0.55} & \multirow{5}{*}{ N.S } \\
\hline & $\mathrm{F} 2$ & 3 & 1.0933 & .09292 & & \\
\hline & $\mathrm{F} 3$ & 5 & 1.4020 & .37090 & & \\
\hline & $\mathrm{F} 4$ & 5 & 1.2600 & .15556 & & \\
\hline & F5 & 4 & 1.2200 & .43688 & & \\
\hline
\end{tabular}

Table (9): Comparison between study patient as regard serum haptoglobin based on stage of fibrosis measured by fibrotest and METAVIR (by non-parametric Kruskal Wallis test):

\begin{tabular}{|c|c|c|c|c|c|c|}
\hline & & $\mathbf{N}$ & Mean & Std. Deviation & p-value & Significance \\
\hline \multirow{5}{*}{$\begin{array}{c}\text { Serum Haptoglobin } \\
(\mathrm{mg} / \mathrm{dl})\end{array}$} & $\mathrm{F} 1$ & 3 & .8500 & .50744 & \multirow{5}{*}{0.043} & \multirow{5}{*}{ S. } \\
\hline & $\mathrm{F} 2$ & 3 & 1.3000 & .26458 & & \\
\hline & F3 & 5 & 1.6320 & .67199 & & \\
\hline & $\mathrm{F} 4$ & 5 & 1.0860 & .37038 & & \\
\hline & F5 & 4 & .5625 & .35556 & & \\
\hline
\end{tabular}


Table (10): Comparison between study patient as regard $\alpha 2$ macroglobulin based on stage of fibrosis measured by fibrotest and METAVIR (by non-parametric Kruskal Wallis test):

\begin{tabular}{|c|c|c|c|c|c|c|}
\hline & & $\mathbf{N}$ & Mean & Std. Deviation & p-value & Significance \\
\hline \multirow{5}{*}{$\underset{(\mathrm{g} / \mathrm{dl})}{\alpha 2 \mathrm{macroglobulin}}$} & F1 & 3 & 1.2733 & .22301 & \multirow{5}{*}{$<0.01$} & \multirow{5}{*}{ H.S. } \\
\hline & $\mathrm{F} 2$ & 3 & 1.4367 & .08083 & & \\
\hline & $\mathrm{F} 3$ & 5 & 2.1980 & .62635 & & \\
\hline & F4 & 5 & 3.0820 & .81711 & & \\
\hline & F5 & 4 & 2.8850 & .74335 & & \\
\hline
\end{tabular}

Table (11): Comparison between study patient as regard CA19-9 based on stage of fibrosis measured by fibrotest and METAVIR (by non-parametric Kruskal Wallis test):

\begin{tabular}{|l|c|c|c|c|c|c|}
\hline & & $\mathbf{N}$ & Mean & Std. Deviation & p-value & \multirow{2}{*}{ Significance } \\
\hline \multirow{3}{*}{ CA19-9 (ml) } & F1 & 3 & 4.633 & 3.5572 & & \\
\cline { 2 - 5 } & F2 & 3 & 5.433 & .7572 & & \multirow{3}{*}{ H.S. } \\
\cline { 2 - 5 } & F3 & 5 & 14.080 & 3.3275 & \\
\cline { 2 - 5 } & F4 & 5 & 22.960 & 3.2532 & & \\
\cline { 2 - 5 } & F5 & 4 & 30.625 & 3.2149 & & \\
\hline
\end{tabular}

Table (12): Comparison between study patient as regard Egy-Score based on stage of fibrosis measured by fibrotest and METAVIR (by non-parametric Kruskal Wallis test):

\begin{tabular}{|c|c|c|c|c|c|c|}
\hline & & N & Mean & Std. Deviation & p-value & Significance \\
\hline \multirow{3}{*}{ Egy-Score } & F1 & 3 & 1.4500966 & .644550207 & & \\
\cline { 2 - 5 } & F2 & 3 & 1.7806083 & .646464492 & \multirow{2}{*}{$<0.01$} & H.S. \\
\cline { 2 - 5 } & F3 & 5 & 3.0229450 & .117803213 & \\
\cline { 2 - 5 } & F4 & 5 & 3.5033658 & .164856593 & \\
\cline { 2 - 5 } & F5 & 4 & 4.5472800 & .371504537 & & \\
\hline
\end{tabular}

\section{Discussion:}

Due to the limitations and the invasive nature of liver biopsy, there has been extensive interest in developing non-invasive tests to measure liver fibrosis ${ }^{(\mathbf{1 1})}$. These are alternatives to liver biopsy that can be used in clinical practice, with benefits in terms of cost, risk, and patient convenience ${ }^{(12)}$. Clinically applicable non-invasive tests include radiological studies, transient elastography (TE), and serum markers.

Most noninvasive tests of liver fibrosis were developed with the aim of discriminating between "insignificant", (F0-F1) by METAVIR and clinically "significant" fibrosis $(\geq F 2)$ by METAVIR or for identifying or excluding established cirrhosis in patients with well compensated chronic liver disease. Both these aims are clinically the most relevant ${ }^{(\mathbf{1 3})}$.

A variety of serologic markers have been evaluated to predict the degree of fibrosis in the liver, and panels have been developed that combine assays of multiple markers to improve predictive ability. The most studied panels are the aspartate aminotransferase (AST) to platelet ratio (APRI), FibroTest/FibroSure, Hepascore, and FibroSpect. Overall, studies of the various panels suggest that they have good ability to differentiate patients with significant fibrosis (F2 to F4) from those without significant fibrosis (F0 to F1) ${ }^{(\mathbf{1 4})}$. 
Table (13): Correlation between different parameters:

\begin{tabular}{|c|c|c|c|c|c|c|c|c|c|c|c|}
\hline & & $\begin{array}{c}\text { Age } \\
\text { (years) }\end{array}$ & $\begin{array}{l}\text { ALT } \\
\text { (IU/dl) }\end{array}$ & $\begin{array}{c}\text { GGT } \\
\text { (IU/dl) }\end{array}$ & $\begin{array}{l}\text { Serum } \\
\text { albumin } \\
(\mathrm{mg} / \mathrm{dl})\end{array}$ & \begin{tabular}{|c|} 
Serum \\
bilirubin \\
$(\mathrm{mg} / \mathrm{dl})$
\end{tabular} & $\begin{array}{c}\text { Platelet } \\
\text { count } \\
(\times 109 / \mathrm{L})\end{array}$ & $\begin{array}{l}\text { apo A1 } \\
(\mathrm{mg} / \mathrm{dl})\end{array}$ & \begin{tabular}{|c|} 
Serum \\
Haptoglobin \\
$(\mathrm{mg} / \mathrm{dl})$
\end{tabular} & $\begin{array}{c}\text { Macroglobulin } \\
(\mathrm{g} / \mathrm{dl})\end{array}$ & $\begin{array}{c}\text { CA19- } \\
9 \\
(\mathrm{ml})\end{array}$ \\
\hline \multirow[t]{2}{*}{ Age (years) } & $\mathrm{r}$ & 1 & .424 & $.499^{*}$ & $-.308-$ & . 158 & $-.485^{*}$ & .213 & .046 & .245 & $.458^{*}$ \\
\hline & p-value & & .063 & .025 & .187 & .505 & .030 & .367 & .846 & .297 & .042 \\
\hline \multirow[t]{2}{*}{ ALT (IU/dl) } & $\mathrm{r}$ & .424 & 1 & .354 & .017 & .055 & $-.314-$ & $-.174-$ & .184 & $-.141-$ & $-.008-$ \\
\hline & p-value & .063 & & 126 & 945 & .817 & .178 & .464 & .437 & .554 & .972 \\
\hline \multirow[t]{2}{*}{ GGT (IU/dl) } & $\mathrm{r}$ & $.499^{*}$ & .354 & 1 & $-.233-$ & .418 & $-.546^{*}$ & $-.092-$ & $-.302-$ & .282 & $.542^{*}$ \\
\hline & p-value & .025 & .126 & & .323 & .067 & .013 & .698 & 196 & .228 & .013 \\
\hline \multirow{2}{*}{$\begin{array}{l}\text { Serum } \\
\text { albumin } \\
(\mathrm{mg} / \mathrm{dl})\end{array}$} & $\mathrm{r}$ & $-.308-$ & .017 & $-.233-$ & 1 & $-.517^{*}$ & .434 & . 109 & .372 & $-.534^{*}$ & $.729^{* * *}$ \\
\hline & p-value & .187 & .945 & .323 & & .020 & .056 & .647 & .106 & .015 & .001 \\
\hline \multirow{2}{*}{$\begin{array}{l}\text { Serum } \\
\text { bilirubin } \\
(\mathrm{mg} / \mathrm{dl})\end{array}$} & $\mathrm{r}$ & .158 & .055 & .418 & $-.517^{*}$ & 1 & $-.357-$ & $-.166-$ & $-.445^{*}$ & $.586^{* *}$ & $.738^{* *}$ \\
\hline & p-value & .505 & .817 & .067 & .020 & & .122 & .485 & .049 & .007 & .001 \\
\hline \multirow{2}{*}{$\begin{array}{l}\text { Platelet count } \\
(\times 109 / \mathrm{L})\end{array}$} & $\mathrm{r}$ & $-.485^{*}$ & $-.314-$ & $-.546^{*}$ & .434 & $-.357-$ & 1 & .351 & .292 & $-.206-$ & $-.535^{*}$ \\
\hline & p-value & .030 & .178 & .013 & .056 & .122 & & .129 & .212 & .383 & .015 \\
\hline \multirow{2}{*}{$\begin{array}{l}\text { apo A1 } \\
(\mathrm{mg} / \mathrm{dl})\end{array}$} & $\mathrm{r}$ & .213 & $-.174-$ & $-.092-$ & .109 & $-.166-$ & .351 & 1 & .345 & .291 & .075 \\
\hline & p-value & .367 & .464 & .698 & .647 & .485 & .129 & & .136 & .214 & .755 \\
\hline \multirow{2}{*}{$\begin{array}{l}\text { Serum } \\
\text { Haptoglobin } \\
(\mathrm{mg} / \mathrm{dl})\end{array}$} & $\mathrm{r}$ & .046 & .184 & $-.302-$ & .372 & $-.445^{*}$ & .292 & .345 & 1 & $-.007-$ & $-.229-$ \\
\hline & $p$-value & .846 & .437 & .196 & .106 & .049 & .212 & .136 & & .978 & .331 \\
\hline \multirow{2}{*}{$\begin{array}{l}\text { Macroglobulin } \\
\text { (g/dl) }\end{array}$} & $\mathrm{r}$ & .245 & $-.141-$ & .282 & $-.534^{*}$ & $.586^{* *}$ & $-.206-$ & .291 & $-.007-$ & 1 & $.830^{* *}$ \\
\hline & p-value & .297 & .554 & .228 & .015 & .007 & .383 & .214 & .978 & & .001 \\
\hline \multirow[t]{2}{*}{ CA19-9 (ml) } & $\mathrm{r}$ & $.458^{*}$ & $-.008-$ & $.542^{*}$ & $-.729^{* * *}$ & $.738^{* *}$ & $-.535^{*}$ & .075 & $-.229-$ & $.830^{* *}$ & 1 \\
\hline & $p$-value & .042 & .972 & .013 & .000 & .000 & .015 & .755 & .331 & .000 & \\
\hline \multirow[t]{2}{*}{ Egy-Score } & $\mathrm{r}$ & $.636^{* *}$ & .202 & $.601^{* *}$ & $-.744^{* *}$ & $.702^{* *}$ & $-.711^{* *}$ & -.006- & $-.274-$ & $.733^{* *}$ & $.936^{* *}$ \\
\hline & $\mathrm{p}$-value & .003 & .394 & .005 & .000 & .001 & .000 & .980 & .243 & .000 & .001 \\
\hline
\end{tabular}

Tumor markers are frequently elevated in patients with chronic liver diseases especially CA 19-9. CA 19-9 can be used as markers for hepatic fibrosis. Both significant and advanced hepatic fibrosis could be predicted by a novel panel of serum biomarkers (Egy-Score) composed of CA 19-9, age, alpha-2macroglobulin, total bilirubin, albumin and platelet count (in a regression equation) with good sensitivity and specificity. Egy-Score can be applied easily in clinical practice to exclude severe hepatic fibrosis/cirrhosis in patients with contraindication for liver biopsy or those who are reluctant to do it, Although this panel needs to be done in validated laboratories, the cost of our score is much cheaper than other wellknown and patented tests such as FibroTest.

Schoniger-Hekele and Muller (15) have reported that tumor markers CA 19-9, CA 125 , and CA 15-3 are increased with stage of hepatic fibrosis and reported that the combined elevation of CA 19-9 and CA 125 is useful for identifying patients with advanced fibrosis or cirrhosis with high specificity.

In our study we aimed at exploring the accuracy of Egy-score in determined the stage of liver fibrosis in patients with chronic liver disease of hepatitis c etiology and comparing it 
with fibrotest result with the gold stander liver biopsy.

Our study included 20 treatment naive patients with chronic liver disease of hepatitis c etiology referred to the outpatient clinics of department of gastroenterologyAnd hepatology, Elhussin hospital Al-Azhar University

We assessed fibrotest (A-2$\operatorname{macroglobulin}(\mathrm{g} / \mathrm{L}), \quad$ haptoglobin $(\mathrm{g} / \mathrm{L})$, apolipoprotein $\quad \mathrm{A} 1(\mathrm{~g} / \mathrm{L}), \quad$ total Bilirubin(umol/L), GGT(IU/L), ALT(IU/L)) and sex, also we assessed Egy-score (albumin $(\mathrm{g} / \mathrm{L}), \quad$ A-2-macroglobulin (g/L), platelet count, total Bilirubin(umol/L), CA19,9 $(\mathrm{U} / \mathrm{ml})$ and age . with comparing the result with each other and with the gold standered liver biopsy result.

Our results revealed a highly significant positive correlation between stage of hepatic fibrosis by METAVIR and alpha-2 macroglobulin.

These findings are concordant with results of Atanasova et al. ${ }^{(16)}$ who found that $\alpha 2$ macroglobulin is quite an informative serum biomarker that reflects liver fibrosis and fibrogenesis in chronic hepatitis C. It can be used in combination with haptoglobin, IgG and AST in scoring systems for non-invasive assessment of liver fibrosis and figrogenesis.

Our results reported a highly significant positive correlation between stage of hepatic fibrosis by METAVIR and GGT

Our results showed a highly significant positive correlation between stage of hepatic fibrosis by METAVIR and total bilirubin

These findings are concordant with results of Cengiz et al. ${ }^{\left({ }^{17)}\right.}$ who reported that association between bilirubin levels and higher surrogate indices of liver fibrosis among participants with $\mathrm{HCV}$ infection. This suggests that bilirubin levels, especially direct bilirubin, were independently associated with an increased risk of increased fibrosis indices.

Our results showed a highly significant positive correlation between stage of hepatic fibrosis by METAVIR and CA19.9.

Our results showed a highly significant positive correlation between stage of hepatic fibrosis by METAVIR and ALT

These findings are concordant with results of Huang et al. ${ }^{(18)}$. Observed that levels of ALT, total bilirubin, alpha 2-macroglobulin and GGT were significantly correlated with the clinical staging of liver fibrosis, and concluded that a combined assessment of these indices might help obtain an accurate diagnosis of liver cirrhosis with less need of pathological biopsy in this population.

Our results showed a highly significant positive correlation between stage of hepatic fibrosis by METAVIR and age.

The results agree with results of Wai $\boldsymbol{e t}$ al. ${ }^{(19)}$, who reported that age was positively associated with severe hepatic fibrosis, and the probability of severe fibrosis increased with increasing values of serum AST, alkaline phosphatase, total bilirubin, and INR.

Our results showed a significant negative correlation between stage of hepatic fibrosis by METAVIR and albumin.

The results also agree with results of Oettl et $\boldsymbol{a l} .{ }^{(20)}$. It may be expected that the reduced concentration of albumin seen in advanced cirrhosis due to impaired synthesis could impact on these clinical sequelae given its multifunctional properties.

Our results showed a significant negative correlation between stage of hepatic fibrosis by METAVIR and haptoglobin.

The results agree with results of Seiichi et al. (21), who reported that the potential usefulness of Fucosylated Haptoglobin and Mac-2 Binding Protein in evaluating liver fibrosis in patients with chronic hepatitis $\mathrm{C}$ who underwent liver biopsy. We demonstrated that the serum levels of both Fucosylated Haptoglobin and Mac-2 Binding Protein were associated with liver fibrosis in patients with chronic hepatitis $\mathrm{C}$, and the combined use of these biomarkers with known fibrosis markers exhibited clinical significance in evaluating liver fibrosis in the chronic hepatitis $\mathrm{C}$ patients.

Our results showed a significant negative correlation between stage of hepatic fibrosis by METAVIR and platelets.

The results agree with results of Giannini et al. (22), which show that Thrombocytopenia (platelet counts $<150,000 / \mu \mathrm{L})$ is a common complication in patients with chronic liver disease (CLD), reported in as many as $76 \%$ of cirrhotic patients.

There was no significant linear correlation between fibrosis stage and Apo A1.

The results disagree with results of Wang et al. ${ }^{(23)}$, which show that ApoA1 is the major protein component of high-density 
lipoprotein in the plasma, and a decrease of ApoA1 has been found in the plasma of patients with hepatic cirrhosis

Our study showed a highly significant positive correlation between stage of hepatic fibrosis by METAVIR and fibrotest.

The results also agree with results of Yakoob et al. (24) study In 107 patients with chronic hepatitis c, levels of six serum biomarkers (alanine aminotransferase, $\gamma$ glutamyl transpeptidase, total bilirubin, haptoglobin, apolipoprotein, $\quad \alpha-2$ macroglobulin) were determined at the time of liver biopsy. Liver biopsy was evaluated by Metavir score for fibrosis and inflammation. Voluntary blood donors $(n=106)$ were taken as controls for the study and the results showed that Fibrosis estimated by Fibrotest was significantly higher in patients compared to control group.

The current study revealed a highly significant positive correlation between stage of hepatic fibrosis by METAVIR and EGYSCOR.

The results also agree with results of Alboraie et al. ${ }^{\left({ }^{(9)}\right.}$ study aimed at exploring the role of elevated serum tumor markers as predictors of the stage of hepatic fibrosis and to combine them with known biomarkers of liver fibrosis to form a new score that can predict different stages of liver fibrosis. We developed a new score (Egy-Score) that can noninvasively predict significant hepatic fibrosis $(\geq$ F2) with $79.3 \%$ accuracy and advanced hepatic fibrosis/cirrhosis ( $\geq$ F3) with $83.7 \%$ accuracy.

Our scores depend mainly on simple routinely used laboratory parameters (total bilirubin, albumin, platelet count) in addition to age and 2 non routine tests (CA 19-9 and Alpha-2-Macroglobulin). Although this panel needs to be done in validated laboratories, the cost of our score is much cheaper than other well-known and patented tests such as FibroTest and the net results of both methods nearly the same.

Egy-Score can be applied easily in clinical practice to exclude severe hepatic fibrosis/cirrhosis in patients with contraindication for liver biopsy or those who are reluctant to do it. Egy-score would need further validation to be regarded as an alternative to liver biopsy.

One should be careful when interpreting elevated levels of tumor markers
CA 19-9 and CA 125 in patients with chronic liver disease as this could be a benign elevation related to hepatic fibrosis and not necessarily due to underlying malignancies.

Limitations of our study includes: elevation of the tumor markers have been associated with cholestasis in liver disease patients and this may give false positive results for our scores ${ }^{(22)}$, also the study was conducted on a small number of patients and it needs validation with a larger number of patients in a prospectively enrolled cohort.

Conclusion

- Accurate assessment of the extent of liver fibrosis is essential for clinical management so as to predict prognosis and therapeutic decision in patients with liver fibrosis but limitations of liver biopsy have given urgency for development of noninvasive diagnostic procedure for liver fibrosis.

- Egy-scor depend mainly on simple routinely used laboratory parameters (total bilirubin, albumin, platelet count) in addition to age and 2 non routine tests (CA 19-9 and Alpha-2-Macroglobulin). Although this panel needs to be done in validated laboratories, the cost of our score is much cheaper than other well-known and patented tests such as FibroTest and the net results of both methods nearly the same.

- Egy-Score can be applied easily in clinical practice to exclude severe hepatic fibrosis/cirrhosis in patients with contraindication for liver biopsy or those who are reluctant to do it. Egy-score would need further validation to be regarded as an alternative to liver biopsy.

\section{Recommendation}

- Physician should be careful when interpreting elevated levels of tumor markers CA 19-9 and CA 125 in patients with chronic liver disease as this could be a benign elevation related to hepatic fibrosis and not necessarily due to underlying malignancies.

- Elevation of the tumor markers such as CA19.9 have been associated with cholestasis in liver disease patients and this may give false positive results for our 
scores which give Limitations to our study

- The study was conducted on a small number of patients and it needs validation with a larger number of patients in a prospectively enrolled cohort to be regarded as an alternative to liver biopsy.

- Our study included only one type of chronic liver disease (HCV) so our results should be validated in other types of chronic liver diseases.

References:

1. Cho SW and Cheong JY. (2007): Clinical application of non-invasive diagnosis for hepatic fibrosis]. Korean J. Hepatol., 13(2): 129-137.

2. Fierbinteanu-Braticevici C, Purcarea $M$ et al. (2009): Non-biopsy methods to determine hepatic fibrosis. J. Med. Life, 2 (4): 401-406.

3. Friedrich-Rust M, Ong M, Martens S et al. (2008): Performance of transient elastography for the staging of liver fibrosis: a meta-analysis. Gastroenterology, 134 (4): 960-974.

4. Callewaert $N$, Van Vlierberghe $H$, Van Hecke A et al. (2004): Noninvasive diagnosis of liver cirrhosis using DNA sequencer-based total serum protein glycomics. Nat. Med., 10:429.

5. Halfon P, Imbert-Bismut $F$, Messous $D$ et al. (2002): A prospective assessment of the inter-laboratory variability of biochemical markers of fibrosis (FibroTest) and activity (ActiTest) in patients with chronic liver disease. Comp. Hepatol., 1:3.

6. Yoon KT, Lim SM, Park JY et al. (2012): Liver stiffness measurement using acoustic radiation force impulse (ARFI) elastography and effect of necroinflammation. Dig. Dis. Sci., 57:1682.

7. Papastergiou V, Emmanuel T, Andrew K et al. (2012): Burroughs. Non-invasive assessment of liver fibrosis. Ann. Gastroenterol., 25(3): 218-231.

8. Elghamry F, Shalaby H, Elshennawy G et al. (2012): Alboraie and Egyptian liver fibrosis study group. Egy-Score predicts severe hepatic fibrosis with high accuracy in Egyptian patients with chronic liver diseases. Gut, 61 (3): 225.
9. Alboraie M, Khairy M, Elsharkawy A, et al. (2014): Egy-score as a noninvasive score for the assessment of hepatic fibrosis in chronic hepatitis C: A preliminary approach. Saudi. J. Gastroenterol., 20:1704.

10. Poynard T, Bruix J, Schiff ER et al. (2013): Improved inflammatory activity with peginterferon alfa- $2 b$ maintenance therapy in non-cirrhotic prior nonresponders: a randomized study. J Hepatol., 2013: 58:452.

11. Lai $M$ and Afdhal NH (2011): Editorial: staging liver fibrosis in hepatitis $\mathrm{C}$ : a challenge for this decade. Am. J. Gastroenterol., 106(12): 2121-2122.

12. Manning DS and Afdhal NH (2088): Diagnosis and quantitation of fibrosis. Gastroenterology, 134(6):1670-81.

13. Strader DB, Wright T, Thomas DL et al. (2004): Diagnosis, management, and treatment of hepatitis C. Hepatology, 39(4):1147-1171.

14. Chou $R$ and Wasson $N$ (2013): Blood tests to diagnose fibrosis or cirrhosis in patients with chronic hepatitis $\mathrm{C}$ virus infection: a systematic review. Ann. Intern. Med., 158:807.

15. Schoniger-Hekele $M$ and Muller $C$ (2006): The combined elevation of tumor markers CA 19-9 and CA 125 in liver disease patients is highly specific for severe liver fibrosis. Dig Dis Sci ., 51:33845.

16. Atanasova E, Martinova F, Jelev D et al. (2015): Alpha-2 macroglobulin is the simplest serum biomarker for liver fibrosis and fibrogenesis in chronic hepatitis C. MedInform., 2: 153-164.

17. Cengiz M, Yilmaz G, Ozenirler S et al. (2014): The association between indirect bilirubin levels and liver fibrosis due to chronic hepatitis $\mathrm{C}$ virus infection. Pathol. Res. Pract., 210:488-493.

18. Huang YW, Yang SS, Fu SC et al. (2014): Increased risk of cirrhosis and its decompensation in chronic hepatitis $\mathrm{C}$ patients with new-onset diabetes: a nationwide cohort study. Hepatology, 60:807.

19. Wai CT, Greenson JK, Fontana RJ et al. (2003): A simple noninvasive index can predict both significant fibrosis and 
cirrhosis in patients with chronic hepatitis C. Hepatology, 38:518.

20. Oettl K, Birner-Gruenberger R, Spindelboeck W et al. (2013): Oxidative albumin damage in chronic liver failure: relation to albumin binding capacity, liver dysfunction and survival. J. Hepatol., 59:978-983.

21. Seiichi T, Tomohide T, Sadaharu I et al. (2016): Evaluation of Fucosylated Haptoglobin and Mac-2 Binding Protein as Serum Biomarkers to Estimate Liver Fibrosis in Patients with Chronic Hepatitis C. https://doi.org/ $\underline{10.1371 / \text { journal.pone. } 0151828}$
22. Giannini E, Borro $P$, Botta $F$ et al. (2000): Cholestasis is the main determinant of abnormal CA 19-9 levels in patients with liver cirrhosis. Int. J. Biol. Markers, 15(3):226-30.

23. Wang Y, Hao J, Liu X et al. (2016): The mechanism of apoliprotein A1 downregulated by Hepatitis B virus. Lipids Health Dis., 15:64.

24. Yakoob R, Bozom IA, Thandassery RB et al. (2015): Noninvasive biomarkers FibroTest and ActiTest versus liver biopsy in chronic hepatitis $\mathrm{C}$ patients: the Middle East experience. Ann. Gastroenterol., Ann Gastroenterol., 28(2): 265-270. 